\title{
Intracranial Hemorrhage in a Pediatric Patient with Chronic Myeloid Leukemia in Chronic Phase: A Case Report
}

\author{
Nobuhisa Takahashi Hideki Sano Kazuhiro Mochizuki \\ Shogo Kobayashi Yoshihiro Ohara Atsushi Kikuta \\ Department of Pediatric Oncology, Fukushima Medical University, Fukushima, Japan
}

\section{Keywords}

Child $\cdot$ Intracranial hemorrhage $\cdot$ Chronic myeloid leukemia in chronic phase

\begin{abstract}
Chronic myeloid leukemia (CML) is an uncommon entity in pediatric patients. CML in chronic phase (CML-CP) has a relatively favorable outcome. Leukostasis occurs in $9.7 \%$ of patients with $\mathrm{CML}$. One of the most serious leukostasis-related complications is intracranial hemorrhage $(\mathrm{ICH})$. However, this is very rare in patients with $\mathrm{CML}-\mathrm{CP}$, and few early mortalities have been reported in CML patients with leukostasis. We report the case of a 14-year-old female patient with CML-CP who developed ICH 8 days after admission. A 14-year-old girl developed symptoms of fatigue and slight fever and was diagnosed with CML-CP. She was treated with imatinib and received low-molecular-weight heparin owing to coagulation abnormalities. However, 6 days later, she developed sensorineural hearing loss, which is a symptom of leukostasis. She received hydroxyurea to reduce her white blood cell (WBC) count, and her treatment was changed from imatinib to nilotinib. The WBC and platelet counts remained unchanged, blast counts did not increase, and mild coagulation abnormality persisted. Eight days after admission, she suddenly lost consciousness and experienced respiratory arrest. Cranial computed tomography revealed multiple $\mathrm{ICH}$ lesions and brain hernia. She received intensive care but was diagnosed with brain death by electroencephalography and died 14 days after hospitalization. $\mathrm{ICH}$ is very rare in patients with $\mathrm{CML}-\mathrm{CP}$; however, patients with leukostasis and coagulation abnormalities can develop severe hemorrhage, even in the chronic phase. Thus, it is necessary to accurately estimate the cause and provide appropriate treatment for these patients.




\section{Introduction}

Chronic myeloid leukemia (CML) is an uncommon entity in children and accounts for $2-3 \%$ of all cases of pediatric leukemias [1]. CML in chronic phase (CML-CP) has a relatively favorable outcome. Suttorp et al. [2] reported an event-free survival rate of $97 \%$ by 18 months in the CML-PAED-II phase III trial. Leukostasis is a pathologic condition caused by hypoxemia due to vascular occlusion in leukemic patients with hyperleukocytosis. Leukostasis occurs in 9.7\% of patients with CML [3]. One of the most serious leukostasis-related complications is intracranial hemorrhage (ICH) [4]. However, this is very rare in patients with CML-CP, and few early mortalities have been reported in CML patients with leukostasis [5]. We report the case of a 14-year-old patient with CML-CP who developed ICH 8 days after admission.

\section{Case Report}

A 14-year-old girl with initial symptoms of fatigue and slight fever was admitted to our hospital. No symptoms of the central nervous system or respiratory system were noted. Physical examination showed that her liver and spleen were palpable at 8 and $10 \mathrm{~cm}$ below their respective costal margins. Her white blood cell (WBC) count was $75.4 \times 10^{4} / \mu \mathrm{L}$ (blasts, $2 \%$; myelocytes, $50 \%$; metamyelocytes, $8 \%$; neutrophils, $29 \%$; monocytes, $2 \%$; lymphocytes, $1 \%$; basophils, $2 \%$ ), her hemoglobin level was $8.5 \mathrm{~g} / \mathrm{dL}$, and her platelet count was $17.2 \times$ $10^{4} / \mu \mathrm{L}$.

Biochemical examination results revealed an elevated lactate dehydrogenase level of $1,579 \mathrm{IU} / \mathrm{L}$ and a soluble interleukin-2 receptor level of $969 \mathrm{IU} / \mathrm{mL}$. No liver dysfunction, renal dysfunction, or electrolyte abnormalities were noted. Coagulation test results were as follows: prothrombin time-international normalized ratio (PT-INR), 1.42 (range, 0.8-1.2); activated partial thromboplastin time (APTT), $43.0 \mathrm{~s}$ (range, 23.0-38.0 s); fibrinogen level, $163 \mathrm{mg} / \mathrm{dL}$ (range, 181-398 mg/dL); D-dimer, $9.4 \mu \mathrm{g} / \mathrm{mL}$ (range, $<1.0 \mu \mathrm{g} / \mathrm{mL}$ ); fibrin/ fibrinogen degradation products (FDP), $26.7 \mu \mathrm{g} / \mathrm{mL}$ (range, $<5.0 \mu \mathrm{g} / \mathrm{mL}$ ); and antithrombin III (AT III) level, 107\% (range, 80-130\%).

No ICH or lung infiltration was observed on contrast-enhanced computed tomography (CT). Bone marrow aspiration showed hypercellularity (nucleated cell count $81.6 \times 10^{4} / \mu \mathrm{L}$ ) but no increased blasts. The chromosomal karyotype was 46XX, t(9:22)(q34:q11.1). In chimeric gene analysis using the novel real-time quantitative polymerase chain reaction

Fig. 1. Cranial computed tomography at the onset of the loss of consciousness and respiratory arrest showing multiple intracranial hemorrhagic legions.

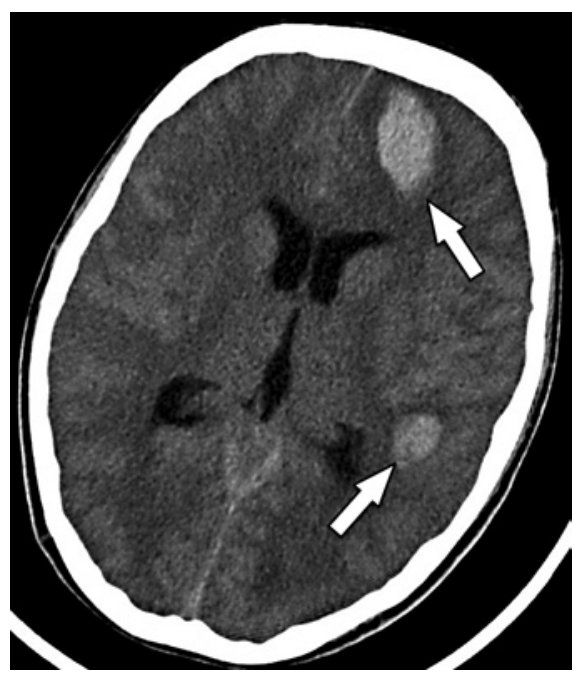




\section{Case Reports in Oncology}

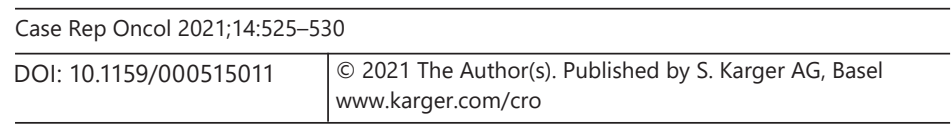

Takahashi et al.: Intracranial Hemorrhage in a Child with Chronic Myeloid Leukemia
Fig. 2. Pure-tone audiometry. Both air conduction and bone conduction thresholds worsened, and there were no significant differences between air and bone conduction thresholds. This patient developed bilateral sensorineural hearing loss.

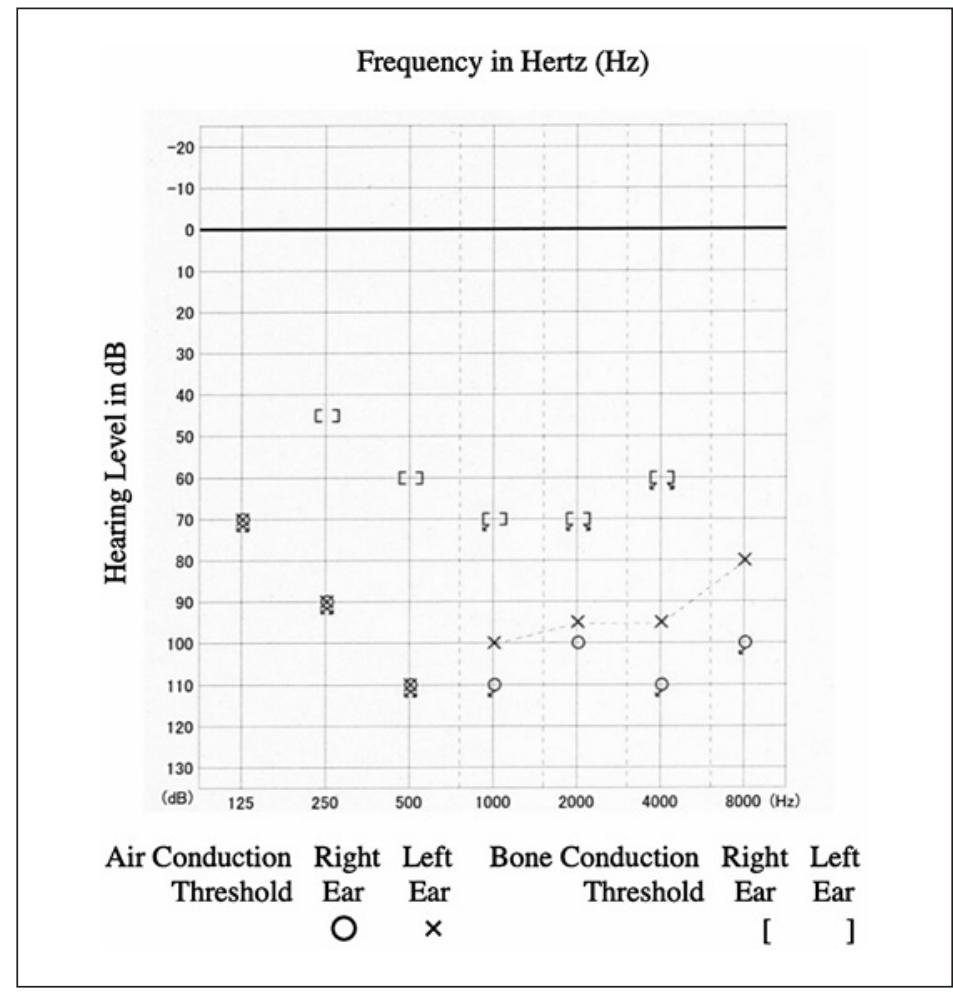

method, a high level of BCR-ABL protein was detected. The BCR-ABL T315I mutation, which indicates imatinib resistance, was not detected. Thus, the patient fulfilled all the 2017 World Health Organization diagnostic criteria for CML-CP.

The patient received hydration, allopurinol, and low-molecular-weight heparin (dalteparin sodium $75 \mathrm{IU} / \mathrm{kg}$ ) after admission to our hospital because we were concerned that her condition would progress to tumor lysis syndrome [6] and disseminated intravascular coagulation (DIC) [7] with the treatment. Her condition was diagnosed as CML-CP 3 days after admission, and imatinib was administered. However, 6 days later, the patient developed sensorineural hearing loss (Fig. 1). We judged this sensorineural hearing loss as a symptom caused by leukostasis, and the patient received hydroxyurea to reduce her WBC count. Her treatment also changed from imatinib to nilotinib. The WBC and platelet counts remained unchanged, blast counts did not increase, and the mild coagulation abnormality persisted.

Eight days after admission, the patient suddenly lost consciousness and experienced respiratory arrest. Cranial CT revealed multiple ICH lesions and brain hernia (Fig. 2). Her WBC count was $73.3 \times 10^{4} / \mu \mathrm{L}$ (blasts, $2 \%$ ), and platelet count was $20.2 \times 10^{4} / \mu \mathrm{L}$. Coagulation test results were as follows: PT-INR, 1.28; APTT, $46.5 \mathrm{~s}$; fibrinogen level, $184 \mathrm{mg} / \mathrm{dL}$; D-dimer, $4.6 \mu \mathrm{g} / \mathrm{mL}$; FDP, $9.2 \mu \mathrm{g} / \mathrm{mL}$; and AT III level, 116\%. The patient received intensive care but was diagnosed with brain death by electroencephalography and died 14 days after hospitalization. An autopsy was not performed because permission could not be obtained from her family (Fig. 3).

\section{Discussion}

Leukostasis is a pathologic condition that is caused by hypoxemia due to vascular occlusion in leukemic patients with hyperleukocytosis. Patients with CML are at risk of developing leukostasis when their WBC count is $>30.0 \times 10^{4} / \mu \mathrm{L}$ and spleen size $>10 \mathrm{~cm}$ [3]; thus, 


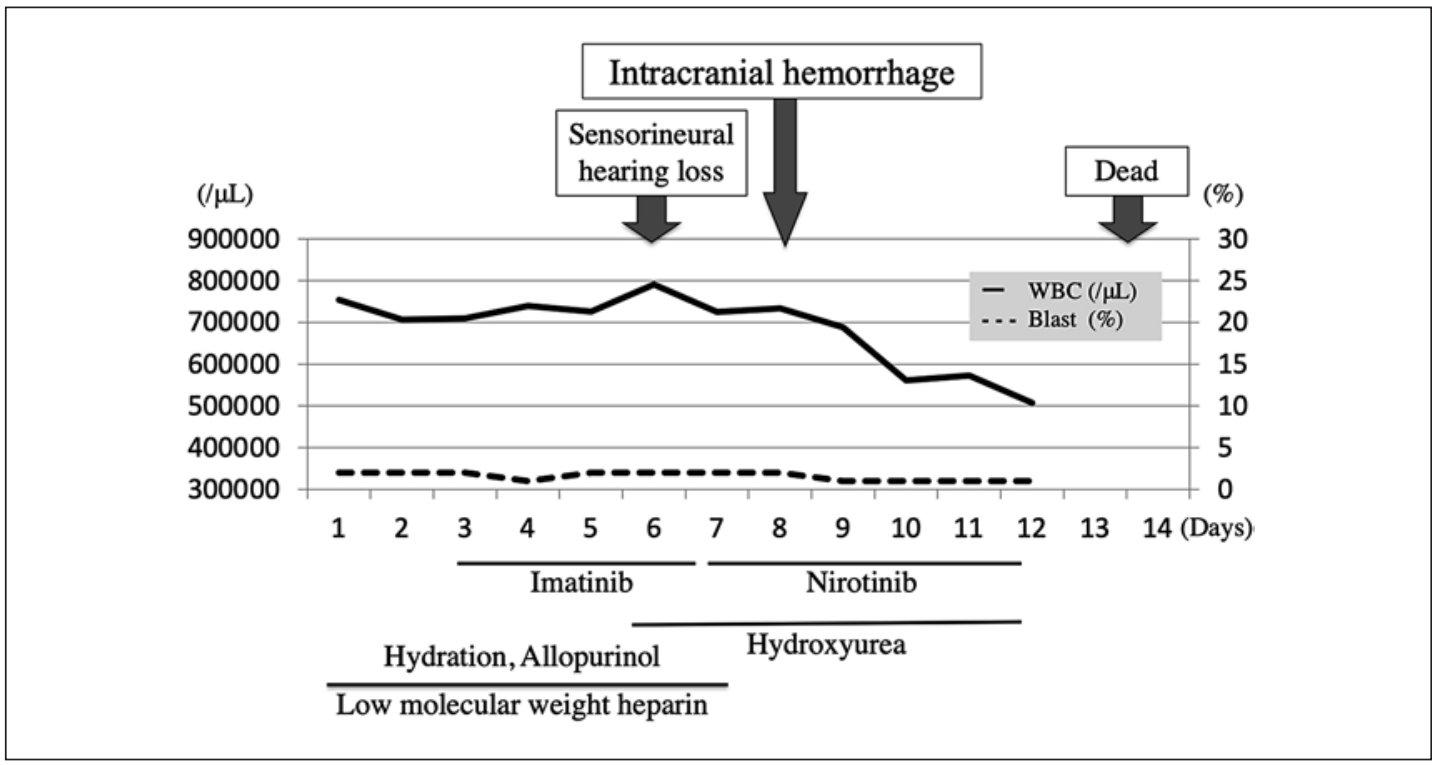

Fig. 3. The patient was treated with imatinib but developed sensorineural hearing loss. She received hydroxyurea, and her treatment was changed from imatinib to nilotinib. However, she suddenly lost consciousness and experienced respiratory arrest. She received intensive care but died 14 days after hospitalization.

it is surmised that our patient was at high risk of developing leukostasis. The main symptoms of leukostasis include respiratory symptoms, such as dyspnea, and central nervous system symptoms, such as headache, visual impairment, sensorineural hearing loss, and tinnitus. Other symptoms include myocardial ischemia and erectile dysfunction [8]. In patients with CML, the main symptoms of leukostasis are ocular symptoms $(13.4 \%)$ and priapism $(6.6 \%)$ [3]. In this patient, the initial symptom of leukostasis was sensorineural hearing loss.

The Japanese Pediatric Leukemia/Lymphoma Study Group's CML Committee reviewed records of 256 Japanese CML pediatric patients aged $<20$ years between 1996 and 2011 at 93 institutes and reported that although 21 of 214 CML-CP patients complained of symptoms caused by leukostasis, there were few early mortalities in CML patients with leukostasis [3]. However, other reports have described that CML-CP patients experience ICH due to leukostasis [5]. Leukostasis symptoms usually occur because of occlusion of the cerebral microvasculature, leading to ICH [9]. In this patient, the main cause of ICH was microvascular occlusion caused by leukostasis. Other reports suggested that coagulation abnormalities such as DIC associated with hyperleukocytosis [10] and acquired von Willebrand disease [11] were the causes of ICH; however, in our patient, the DIC score defined using the diagnostic criteria for DIC of the Japanese Society on Thrombosis and Hemostasis (2017 edition) was 3, and there was no exacerbation of a coagulation abnormality. However, few patients with CML-CP develop fatal bleeding. Nonetheless, some CML cases with severe bleeding caused by multiple factors, including platelet dysfunction [12], fibrinolytic activation [13], and side effects of tyrosine kinase inhibitors [14], have been reported. We used low-molecular-weight heparin because we were worried that her condition would progress to DIC with the treatment [7]. However, the use of low-molecular-weight heparin may have affected the coagulation function of this patient. The use of anticoagulants in such patients should be considered thoroughly.

Our patient received hydration, allopurinol, low-molecular-weight heparin, and tyrosine kinase inhibitor after admission to our hospital because her condition was diagnosed as CML-CP, and the severity of her leukostasis was moderate (Novotny grading system grade 2 
[15]). However, she developed severe ICH and died. It is possible that the reason for her severe cerebral hemorrhage was not only leukostasis but also the complicated coagulopathy in CML. Thus, further investigations for coagulopathy should have been performed. However, her condition progressed so rapidly that complicated coagulopathy could not be scrutinized.

\section{Conclusion}

ICH is very rare in patients with CML-CP. However, in the case of CML with leukostasis and coagulopathy, abnormal coagulation is caused by various pathologic conditions. Thus, it is necessary to accurately estimate the cause and provide appropriate treatment for these patients.

\section{Statement of Ethics}

Written informed consent was obtained from the patient's parents for publication of this case report and any accompanying images.

\section{Conflict of Interest Statement}

The authors have no conflicts of interest to declare.

\section{Funding Sources}

There were no funding sources.

\section{Author Contributions}

N. Takahashi drafted the initial manuscript. K. Mochizuki, S. Kobayashi, and Y. Ohara were involved in the care of the patient. A. Kikuta reviewed and revised the manuscript and approved the final manuscript as submitted. H. Sano supervised each step of the writing of the manuscript. All authors read and approved the final manuscript and agree to be accountable for all aspects of the work.

\section{References}

1 Hijiya N, Schultz KR, Metzler M, Millot F, Suttorp M. Pediatric chronic myeloid leukemia is a unique disease that requires a different approach. Blood. 2016;127(4):392-9.

2 Suttorp M, Schulze P, Glauche I, Göhring G, von Neuhoff N, Metzler M, et al. Front-line imatinib treatment in children and adolescents with chronic myeloid leukemia: results from a phase III trial. Leukemia. 2018;32: 1657-69.

3 Kurosawa H, Tanizawa A, Tono C, Watanabe A, Shima H, Ito M, et al. Leukostasis in children and adolescents with chronic myeloid leukemia: Japanese Pediatric Leukemia/Lymphoma Study Group. Pediatr Blood Cancer. 2016;63(3):406-11.

4 Kouzuki K, Umeda K, Saida S, Kato I, Hiramatsu H, Funaki T, et al. Sudden intracranial hemorrhage in a patient with atypical chronic myeloid leukemia in chronic phase. J Pediatr Hematol Oncol. 2018;40(8):e553-6.

5 Wang H, Cao F, Li J, Sun K, Jin J, Wang M. Intracerebral hemorrhage as the initial presentation of chronic myeloid leukemia: a case report and review of the literature. Front Neurol. 2020;11:571576.

\section{Karger's}


6 Hua J, Iwaki Y, Inoue M, Hagihara M. Tumor lysis syndrome soon after treatment with hydroxyurea followed by nilotinib in two patients with chronic-phase chronic myelogenous leukemia. Int J Hematol. 2013;98(2): 243-6.

7 Martí-Carvajal AJ, Anand V, Solà I. Treatment for disseminated intravascular coagulation in patients with acute and chronic leukemia. Cochrane Database Syst Rev. 2015;2015(6):CD008562.

8 Rollig C, Ehninger G. How I treat hyperleukocytosis in acute myeloid leukemia. Blood. 2015;125(21):3246-52.

9 Lichtman MA, Rowe JM. Hyperleukocytic leukemias: rheological, clinical, and therapeutic considerations. Blood. 1982;60(2):279-83.

10 Giammarco S, Chiusolo P, Piccirillo N, Di Giovanni A, Metafuni E, Laurenti L, et al. Hyperleukocytosis and leukostasis: management of a medical emergency. Expert Rev Hematol. 2017;10(2):147-54.

11 Assem MM, Aly MAM, Salam IA, Shaheen MH, Zuhair MN, Saleh M, et al. Acquired von Willebrand disease in hematologic malignancies at the National Cancer Institute (NCI) Egypt. J Egypt Natl Cancer Inst. 2000;12: 117-23.

12 Akay OM, Mutlu F, Gülbaş Z. Platelet dysfunction in patients with chronic myeloid leukemia: does imatinib mesylate improve it? Turk J Haematol. 2016;33(2):127-30.

13 Rość D, Kremplewska-Nalezyta E, Gadomska G, Bielis L. [Hemostatic disturbances in chronic myeloid leukemia]. Wiad Lek. 2007;60(3-4):138-42.

14 Kim MS, Lee DH, Lee YR, Kim DK, Bae SH, Hwang JY, et al. A case of subdural hematoma in patient with chronic myeloid leukemia treated with high-dose imatinib mesylate. Korean J Hematol. 2010;45(1):73-5.

15 Novotny JR, Müller-Beissenhirtz H, Herget-Rosenthal S, Kribben A, Dührsen U. Grading of symptoms in hyperleukocytic leukaemia: a clinical model for the role of different blast types and promyelocytes in the development of leukostasis syndrome. Eur J Haematol. 2005;74(6):501-10. 